\title{
Correlation of spherical nanoindentation stress-strain curves to simple compression stress- strain curves for elastic-plastic isotropic materials using finite element models
}

\author{
Dipen K. Patel and Surya R. Kalidindi \\ Woodruff School of Mechanical Engineering, Georgia Institute of Technology, Atlanta, GA 30332, USA
}

\begin{abstract}
The stress-strain fields realized in spherical indentation tests are highly heterogeneous, and present a significant challenge to the recovery of bulk stress-strain responses such as those measured in simple compression tests performed on samples with a uniform cross section in the gauge section. In this paper, we critically explore the correlations between indentation stressstrain curves and the simple compression stress-strain curves using the finite element model of indentation as a surrogate for the actual experiment. The central advantage of using a finite element model is that it allows us to critically explore the sensitivity of various assumptions or values of parameters or other choices made in the analyses protocols on the extracted results. Based on this study, a general protocol has been established to reliably recover the uniaxial stress-strain response directly from the indentation stress-strain curve for isotropic elastic-plastic materials. The protocols developed are validated for a range of hardening behaviors.
\end{abstract}

Keywords: Scaling relationship in spherical indentation, Uniaxial response, Isotropic plasticity, Finite element simulation, Constraint factor 


\section{Introduction}

Bulk mechanical properties of materials are typically measured under uniaxial loading conditions such as simple tension or simple compression [1]. However, testing methods for extracting the local properties from exceedingly small subvolumes of a sample are still in developmental stages [2-5]. Knowledge of local mechanical responses is critical for the formulation and validation of physics-based multiscale materials models [6-8]. Since the number of distinct microscale constituents (i.e., local states capturing variations in local chemistry, structure, and process history) of interest in most advanced materials is extremely large, it is highly desirable to have high throughput protocols for characterizing the local mechanical responses in hierarchical material systems.

Recent developments in instrumented indentation techniques [9-17] have resulted in the ability to probe reliably and consistently the mechanical properties of materials at the microscale. More specifically, it has been demonstrated that it is possible to extract suitably normalized indentation stress-strain (ISS) curves that display an initial linear elastic segment followed by a clear transition to a plastic response. Consequently, indentation methods have attracted considerable attention. However, relating these ISS curves to stress-strain responses measured in the conventional simple compression tests remains a significant challenge.

The central issues in the extraction of ISS curves and their correlation to simple compression stress-strain curves revolve around the definitions of the indentation stress and indentation strain measures and their correspondence with stress and strain measures used in simple compression tests. Indentation stress and strain definitions generally stem from Hertz's theory [18] for frictionless contact between two isotropic elastic solids with spherical surfaces, which may be described as 


$$
P=\frac{4}{3} E_{e f f} R_{e f f}^{\frac{1}{2}} h_{e}^{\frac{3}{2}}, \quad a=\sqrt{R_{e f f} h_{e}}
$$

where $a$ is the contact radius at the indentation load, $P$ and $h_{e}$ is the elastic penetration depth. $R_{\text {eff }}$ and $E_{\text {eff }}$ denotes the effective radius and the effective modulus of the indenter and the specimen system. In order to convert the measured load-displacement data into ISS curve, one may define the indentation stress and indentation strain such that Hertz's theory, Eq. (1), transforms into a linear relationship as

$$
\sigma_{\text {ind }}=\frac{4 E_{e f f}}{3 \pi} \varepsilon_{\text {ind }}, \quad \sigma_{\text {ind }}=\frac{P}{\pi a^{2}}, \quad \varepsilon_{\text {ind }}=\frac{a}{R_{e f f}}
$$

Eq. (2) has prompted many researchers to adopt some variant of $a / R_{\text {eff }}$ as a measure of indentation strain for the more general case of elastic-plastic indentation. In this regard, it is important to note that Eq. (2) is strictly valid only for elastic indentations. As a specific example, a majority of the studies in the literature [10, 19-22] have employed the definition of indentation strain as $a / R_{i}$ for elastic-plastic indentation. This definition of indentation strain as $a / R_{i}$ lacks any physical interpretation as a measure of strain. This is because strain should be fundamentally interpreted as a ratio of the change in length over the initial length of a selected line segment in region of interest in the sample.

Over the past decade, various studies [23-28] have adopted the indentation stress and strain measures described above to extract ISS curves and correlate them to the uniaxial stress-strain (USS) responses. One of the earliest studies aimed at correlating spherical indentation responses to the uniaxial tests is attributed to Tabor [29]. Tabor assumes that the mean contact pressure (load divided by the projected area of the residual impression; consistent with $\sigma_{\text {ind }}$ in Eq. (2)) is 
proportional to the "representative" flow stress of the deforming material and the corresponding "representative" strain is proportional to $a / R_{i}$ (variant of the $\varepsilon_{i n d}$ in Eq. (2)). Tabor was able to empirically correlate experimentally measured values of indentation flow stresses and strains on annealed copper and mild steel with a range of spherical indenter tip radii to the stress-strain measurements in uniaxial stress conditions on the same metals. Specifically, Tabor demonstrated that the mean contact pressure $\left(P_{m}\right)$ scaled by a factor of 2.8 when plotted against $0.2 a / R_{i}$ produces an excellent match with the simple compression stress-strain curves. [29]. The factor of 2.8 is defined as the "constraint" factor to capture the effect of the higher hydrostatic stress inherent to the indentation test. As mentioned earlier, and despite the remarkable agreement seen in Tabor's experiment, the definition of the indentation strain based on $a / R_{i}$ holds no known physical significance. It is also important to note that in Tabor's approach, contact radius is estimated by measuring the residual impression on the unloaded sample. This definition is inconsistent with the definition used in Hertz's theory [18], which is based on the contact radius in the loaded configuration. Tabor's original approach is also very effort consuming as each indentation test produces only one data point in the plastic regime of the ISS curve.

The classical experiments of Tabor have stimulated numerous theoretical studies aimed at correlating the elastic-plastic stress-strain responses in indentation and simple compression. The theoretical treatment for the perfectly-plastic deformation imposed by a rigid frictionless indenter was explored by Ishlinsky [30] and later by Hill [31] using slip-line field approach assuming plane strain deformation (note that the real deformation mode in indentation is far from this assumption). Ishlinsky [30] performed slip-line field analysis of a spherical contact and reported that the contact pressure for a perfectly plastic contact (no hardening) is between 2.61 and 2.84 . Subsequently, Hill [31] applied the slip-line field theory to the rigid plastic deformation of a flat 
sample with a wedge indenter, produces the widely cited result that the average pressure under the (wedge) indenter being approximately three times the flow stress in a uniaxial test. It should be noted that the imposed plane-strain boundary conditions in these theoretical approaches naturally yields an upper bound in determining the constraints factor [32].

An alternative approach to the analysis of an elastic-plastic indentation was suggested by Bishop et al. [33], and was further developed extensively by Johnson [34]. The spherical cavity model proposed by Johnson assumes that the surface of the indenter in contact is encased in a hemi-spherical core that essentially comprises both the rigid indenter and the surrounding material. The core is assumed to be in a hydrostatic stress state (i.e., the core acts as an inflating spherical cavity). Outside the core, it is assumed that the stress and displacement are radially symmetric, same as in an infinite elastic perfectly-plastic body containing a spherical cavity under pressure. The stress and displacement fields are computed invoking two conditions at the interface between the core and the elastic-plastic zone [35]: (i) the hydrostatic pressure in the core must be equal the radial component of stress in the external zone, and (ii) neglecting the compressibility in the core, the displacement of points lying on the interface during penetration must accommodate the volume of material displaced by the indenter. The spherical cavity model of indentation predicts that the mean pressure at initial yield (deviation from Hertz theory) is 1.1 times the uniaxial flow stress, and at the fully plastic state, it would reach around 3.0 times the uniaxial flow stress. Although the spherical cavity model allows imposing large plastic deformation on the sample, it neglects the upheaval or "pile-up" behavior of the material around an indenter. Also, the model assumes uniform expansion of the material around the core as in the case of spherical pressurized void in an infinite elastic-plastic space. These assumptions severely limit the utility of this approach. 
In practice, it is very difficult to compute theoretically the contact stresses in an elasticplastic indentation, because the shape and the size of the elastic-plastic boundary cannot be captured adequately in idealized simple geometries. This has led to the development of various numerical methods to the indentation simulation problem. One of the first numerical models for spherical indentation was established by Hill [25] using the infinitesimal deformation theory of plasticity. In this model, Hill invokes geometrical-similarity in the scaling of contact variables as a function of penetration depth with the spherical indenter approximated by a paraboloid of revolution. Hill's model is in excellent agreement with Tabor's findings that the representative indentation strain is $0.2 a / R_{i}$ and that the average pressure is 2.8 times the uniaxial flow stress in tension. It should be noted that Hill's model makes the following simplifying assumptions: (i) the constitutive behavior of the indented half-space is governed by a simple power-law between suitable measures of stress and strain, and the elastic contribution to the deformation is neglected, (ii) the contact geometry remains constant throughout the indentation, and (iii) the diameter of the indenter is very large compared to the indentation depth leading to infinitesimal plastic strain imposed on the material. In spite of these simplifying assumptions, it is remarkable that the predictions of the numerical model are in excellent agreement with Tabor's experiments.

In an effort to extend the applicability of Hill's model [31] while capturing the complex heterogeneous deformation field under the indenter, several recent studies have resorted to finite element models [27, 28, 36, 37]. A majority of these studies report a value of around 3.0 for the constraint factor for an elastic-perfectly plastic response. However, the ISS curves produced by these models show unusually large elastic-plastic transitions with high levels of apparent strain hardening (note that the materials constitutive behavior was assumed to be elastic-perfectly plastic in these models). In a recent study Donohue et al. [38] pointed out that these abnormal 
features of the ISS curves arise because of the use of specific definitions of the contact radius and the indentation strain measures that are completely inconsistent with Hertz's theory. In this regard, it is important to specifically note that the modern indenters are capable of providing continuous estimates of the contact radius throughout the entire elastic-plastic loading segment (recall that Tabor had to fully unload a sample to measure the contact radius from the residual indentation). This is typically accomplished using the continuous stiffness measurements [9, 11, 14-16, 39] together with some variant of the Hertz's theory (Eq. (1)). In light of these recent advances in instrumentation, it is imperative that all parameters (e.g., contact radius, indentation strain measure) utilized in the conversion of the load-displacement curves into ISS curves are fully consistent with Hertz's theory.

A number of other approaches in literature for the recovery of the USS response from the indentation experiments have employed inverse solution methodologies. These approaches utilize sophisticated optimization strategies to minimize a suitably defined difference (i.e., error metric) between the measured load-displacement curve and the corresponding prediction from the finite element simulation by adjusting the material's constitutive law defined usually as its USS response [40-45]. The central deficiency of this approach stems from the fact that the entire elastic-plastic transition occurs over a very short regime in the load-displacement curve. Since it is very difficult to pinpoint this regime exactly in the measured load-displacement curve, it is very difficult to extract reliable properties consistently with such inverse solution methodologies. Indeed, transforming load-displacement curve to ISS curves addresses this problem and provides a much improved opportunity for extracting reliable uniaxial stress-strain curves.

Indentation techniques have also been employed to characterize the slip system level mechanical properties at the scale of individual grains [46] in a polycrystalline sample. Once 
again finite element simulations employing suitable physics-based constitutive models were developed and employed in these studies to capture the heterogeneous deformation behavior [47] and extract the relevant properties of interest by matching the overall load-displacement predictions from the model with the corresponding experimental measurements. Examples of these efforts have included the extraction of single crystal elastic stiffness parameters [48], initial slip-hardening rate [49], and average slip resistance values [50, 51]. These investigations were mainly focused on capturing the orientation dependence of single crystal anisotropic properties using indentation techniques $[52,53]$. However, it is important to establish the correlation between the uniaxial response and the indentation response for the simpler case of isotropic elastic-plastic material behavior before considering the more complex anisotropic case.

In this paper, we restrict our attention to materials exhibiting isotropic elastic-plastic response and demonstrate that the ISS curves extracted using the recently developed spherical indentation protocols $[12,13,17,38]$ can be directly correlated to the USS response via simple scaling factors. These scaling factors are established and validated in this study using a twodimensional finite element model for a class of isotropic elastic-plastic materials obeying the $J_{2}$ flow theories [54] with linear or saturation type hardening laws. The similarities and differences between these scaling factors and the ones originally used by Tabor are discussed in detail.

\section{FE simulation of spherical indentation}

As noted earlier, we will employ a finite element (FE) model of spherical indentation as a surrogate for the experiment in exploring the correlations between ISS and USS responses. For this purpose, we have utilized the commercial finite element code ABAQUS [55]. The FE model developed for this study is shown in Figure 1a, and is comprised of two axisymmetric twodimensional isotropic bodies: (i) an elastic-plastic deformable sample with an initially flat 
surface, and (ii) a rigid hemi-spherical indenter. Four-noded, bilinear, two-dimensional axisymmetric continuum elements (CAX4 in ABAQUS) are used to model the sample in this study. The size of the sample mesh was selected as $20 \mu \mathrm{m} \times 20 \mu \mathrm{m}$ to ensure that it is much larger than the primary indentation zone. The top surface of the indenter is constrained to remain planar, and is allowed to move only normal to the indentation surface. The sample is constrained from moving in the $\mathrm{x}$-direction along the axis of symmetry and along y-direction (indentation direction) at the bottom surface. The sample is discretized into five regions (as shown in Fig. 1a.) to achieve highest mesh density in the primary indentation zone (region in the sample with the highest localized deformation).

In the simulations, a vertical displacement boundary condition (along y-direction) is imposed on the node at the center of the indenter which is tied rigidly to entire surface of the indenter. Surface-to-surface contact definition is used to avoid any concentrated force buildup at individual nodes at initial point of contact. Detachment of the two surfaces is allowed to simulate loading-unloading response. The FE model is first validated for purely elastic indentations by comparing the predicted load-displacement curve to predictions from Hertz's theory (Eq. (1)). Figure $1 \mathrm{~b}$ demonstrates excellent accuracy of the FE indentation model for purely elastic indentations.

The load-displacement history extracted from the FE model in multiple elastic-plastic loading-unloading cycles [38] are then used to extract elastic-plastic ISS curves comparable to those extracted from experiments $[12,13]$. For this purpose, we have adopted the following definitions of indentation stress and indentation strain [56]: 


$$
\sigma_{\text {ind }}=E_{\text {eff }} \varepsilon_{\text {ind }}, \quad \sigma_{\text {ind }}=\frac{P}{\pi a^{2}}, \quad \varepsilon_{\text {ind }}=\frac{4}{3 \pi} \frac{h_{t}}{a} \approx \frac{h_{t}}{2.4 a}
$$

Note that Eq. (3) is essentially a rearrangement of Hertz's theory expressed in Eq. (1). This particular rearrangement suggests the use of the ratio $h_{t} / a$ as the definition of indentation strain. The pre-multiplier, $4 / 3 \pi$ in the definition of indentation strain was justified by noting that $2.4 a$ corresponds to the depth of the indentation zone [38]. This definition of indentation strain allows interpretation of strain measure in the classical sense as the change in length per unit length by idealizing the deformation under indenter as being equivalent to compressing by $h_{t}$ (total average indentation depth) a cylindrical sample of radius $a$ and height $2.4 a$.

The protocols needed to extract ISS curves from the FE model have been described previously by Donohue et al. [38]. A central feature of these protocols is that contact radius is estimated from a partial unloading segment which is purely elastic and follows Hertz's theory. Since each unload leads to an estimation of one value of contact radius (in much the same way Tabor did in his experiments), it becomes necessary to conduct a very large number of loadunload steps (as shown in Figure 2) in the FE simulation to determine accurately the evolution of the effective radius and the residual depth in the sample.

\section{Extracting Scaling Relationship between ISS and USS Response}

This work is aimed at developing robust protocols for recovering the USS curves from the ISS curves. As discussed earlier, this requires the estimation of suitable scaling factors between uniaxial and indentation stresses as well as between uniaxial and indentation strains. Furthermore, we focus on our attention in this study to isotropic elastic-plastic materials, where the plastic response is described by the $J_{2}$ flow theory [54]. 
Our strategy for addressing this challenge involves two steps. In the first step, we focus on the scaling factor between uniaxial and the indentation stress through a consideration of the elastic-perfectly plastic indentation response. Because there is no hardening in this case, we expect the ISS curves to plateau at large strains. As a result, we should be able to extract a simple ratio between the plateau stresses in USS and ISS curves.

In the second step, we focus on the scaling factors for uniaxial and indentation strains. In fact, as soon as we establish a scaling factor for the stress (described in the first step above), the scaling factor for the elastic indentation strain can be extracted by matching the ISS curve obtained using Hertz's theory (Eq. 3) with the corresponding USS curve (note that for elasticity both these curves are linear). Therefore, we only need to focus on the scaling factor for indentation plastic strain. This can be established from a consideration of indentation response of materials exhibiting linear hardening.

Our central hypothesis is that the scaling factors established using the protocols described above are not strongly affected by the actual hardening behavior exhibited by the sample material. We critically evaluate our hypothesis by applying the same scaling factors to a sample material exhibiting a completely different hardening response, namely the saturation-type hardening laws. The calibration and validation of these scaling factors are described next.

\subsection{Scaling Factor for Indentation Stress}

Table 1 summarizes the material properties used in this study for the elastic-perfectly plastic sample. These cover the typical range of properties exhibited by advanced metals of interest to structural applications. The corresponding ISS curves are generated from the finite element model following the protocols described above (cf. [38]). 
These ISS curves obtained using the FE model and elastic-perfectly plastic material response are shown in Figure 3, and show three clearly distinguishable regimes: (i) an initial elastic regime where the indentation stress evolves linearly with the indentation strain, (ii) an elasticplastic transition regime that exhibits apparent strain hardening (recall that there is no real hardening of the material in the elastic-perfectly plastic constitutive description) attributed to the transformation of the indentation zone from being dominated by elasticity to one dominated by plasticity, and (iii) a post-yield regime exhibiting the expected perfectly plastic response.

Based on the linear elastic solution for stress fields beneath the spherical indenter, initiation of plastic flow following the von-Mises criterion is predicted at an indentation stress of $1.07 \sigma_{\mathrm{y}}$ [35]. However, it is important to note that the deviation from elasticity on the ISS happens after a substantial amount of plastic deformation has been realized by the sample as seen in Figure 4 . The elastic-plastic transition is marked by the fact that the stress fields in the indentation zone have to change from those dominated by elasticity in the first regime to those dominated by plasticity in the final regime. More specifically, the plastic zone underneath the indenter is initially fully surrounded by elastically deforming material. This initial plasticity is therefore heavily constrained until the plastic zone has grown to reach the sample surface (see Figure 4). At that point, plasticity occurs relatively easily and this marks the onset of the third regime of a perfectly plastic response in the ISS curves seen in Figs. 3 and 4. In particular, this transition is most clearly seen through the contour plots of equivalent plastic strain (PEEQ) fields at selected points on the ISS curve in Fig. 4. This elastic-plastic transition poses a significant challenge in our efforts to establish a useful correlation between ISS curves and the USS curves.

It should be noted that the ISS curves in Figure 3 exhibit some degree of roughness. This, in our opinion, is a consequence of the many numerical computations involved both in the FE 
simulations (i.e., mesh design, mesh discretization) and the estimation of the contact radius from the analyses of the unloading segments. Our many trials in these simulations have indicated that these numerical oscillations can be alleviated by increasing the mesh density inside the constantly evolving primary indentation zone. However, this improvement is relatively small (and mostly inconsequential for the present study) and incurs a prohibitively high computational cost.

As mentioned earlier, the ability to extract meaningful ISS curves is critical to our goals of extracting a reliable scaling factor between the uniaxial and indentation stresses. This scaling factor is simply the ratio of the indentation stress and the uniaxial stress in the plateau regions of these stress-strain curves. Based on the ISS curves shown in Figure 3, this ratio was observed to be 2.2. The correspondence between the scaled ISS curve and the USS curve for the soft material is shown in Figure 5 (a similar correspondence was observed for the stiff material as well). In Figure 5, the indentation strain was scaled by 2.0 , which is established simply using Hertz's theory (i.e., matching Eqs. (1) and (3)). In other words, this scaling factor ensures that the elastic portions of the scaled ISS and USS curves would match. However, as mentioned earlier, the plastic strain in the indentation and the uniaxial tests are unlikely to be related by the same factor as the corresponding elastic strains; this will be explored in detail in later case studies that invoke strain hardening.

The stress scaling factor (also called constraint factor) of 2.2 is indeed significantly lower than the previously reported numbers (e.g., the factor of 2.8 reported by Tabor [57] and Hill [25, $31,54])$. The difference is directly attributed to the differences in the definition of the contact radius used in the extraction of the ISS curves. More specifically, the contact radius used in prior studies was either based on the residual indentation left after complete unloading or is defined 
based on geometrical relationship between the indenter and the sample. In a recent paper [38], we have demonstrated these choices have a significant effect on the estimated values of the contact radius and the subsequent computation of the indentation stress. In the same paper, we have demonstrated that the protocol used in this work provides the most meaningful ISS curves.

As noted earlier, the elastic-plastic transition in the ISS curve is significant in extent and poses a significant challenge in our efforts to correlate the ISS and USS curves. We will revisit this issue later.

\subsection{Scaling Factor for Indentation Plastic Strain}

As noted earlier, it is necessary to examine the ISS curves for materials exhibiting hardening to extract a scaling relationship between the indentation plastic strain and the uniaxial plastic strain. For this purpose, FE indentation simulations were performed for the soft material (see Table 1) with different levels of constant strain hardening rates, $h_{o}$. In other words, the uniaxial stress-strain behavior of the sample in these simulations is defined to be bi-linear: the first linear segment reflects the purely elastic behavior, and the second linear segment with a slope of $h_{o}$ reflects a regime of constant strain hardening rate. This bilinear stress-strain description (in the uniaxial stress mode) is provided as input to the FE indentation simulations. The corresponding ISS curves obtained using the protocols described earlier are shown in Figure 6.

It is seen that the ISS curves in the plastic regime exhibit a linear hardening response, except for some oscillations that are fairly characteristic of the numerical instabilities involved in these simulations (as discussed earlier). The fact that the ISS curves reflect a linear hardening response lends additional support to the validity of the analyses protocols described and employed in this work. 
The results presented in Figure 6 also suggest that it should be possible to establish a simple scaling factor between the indentation plastic strain and the uniaxial plastic strain. There is, however, no reason to expect that this scaling factor would be the same as the one introduced earlier for the elastic strain. The following set of equations is formulated to describe this equivalence:

$$
\begin{aligned}
& \varepsilon_{\text {ind }}=\varepsilon_{\text {ind }}^{e}+\varepsilon_{i n d}^{p}=\frac{\sigma_{i n d}}{E_{\text {eff }}}+\varepsilon_{\text {ind }}^{p} \\
& \varepsilon_{s c}=\frac{\varepsilon_{i n d}^{e}}{2.0}+\frac{\varepsilon_{i n d}^{p}}{\alpha}
\end{aligned}
$$

In Eqs. (4) and (5), $\varepsilon_{s c}$ denotes the equivalent simple compression strain corresponding to $\varepsilon_{\text {ind }}$ and the superscripts $e$ and $p$ refer to the elastic and plastic components, respectively. The value of the scaling factor $\alpha$ was established as $\sim 1.3$ by matching the ISS and USS curves for all the FE simulations conducted in this work imposing linear hardening. It is noted here that the scaling factor $\alpha$ established in this study is also the same as the scaling factor for the ratio between the hardening rates in ISS and USS responses reported previously by Donohue et al. [38] and confirmed once again in this study. The corresponding scaled ISS curves are compared with the USS curves in Figure 7. It is seen that there is excellent agreement between these curves, except in the elastic-plastic transition regime. As noted earlier, it is very difficult to make a correspondence in this transition regime and we shall revisit this issue later in this paper. 


\section{Validation of the Scaling Relationships between ISS and USS Curves}

As a critical evaluation of the protocols established in the previous section, we examine a material constitutive behavior where the plastic response is described by power-law hardening described as

$$
\sigma=\sigma_{y}+K\left(\varepsilon^{p}\right)^{n}
$$

where $K$ and $n$ are the strength coefficient and the strain hardening exponent. For the present case study, we choose two distinct sets of material parameters with different strength coefficients and strain hardening exponents to describe the uniaxial stress-strain behavior of the sample. The material parameters selected are the ones reported for Tantalum $(n=0.5$ and $K=562 \mathrm{MPa})$ and Aluminum ( $n=0.2$ and $K=180 \mathrm{MPa}$ ) [58]. These power-law descriptions were provided as input to the FE indentation simulations.

The ISS and USS curves for the selected materials are compared in Figure 8. It is seen that scaling protocols described earlier are highly applicable to the both high and low hardening behaviors. This result is significant as the scaling factors were extracted from two very simple hardening laws: (i) perfectly plastic (non-hardening), and (ii) linear hardening, and are observed to provide excellent correspondence in the case of the much more complex saturation-type hardening law used in the validation example. Indeed, the excellent agreement between the scaled ISS and the USS responses, using the scaling factors from the simpler hardening laws, attest clearly to their general applicability to a range of materials behaviors of interest in actual practice. 


\section{Indentation Yield Strength and Correlation to Uniaxial Yield Strength}

As clearly evident from the case studies presented so far, reliable identification of the indentation yield strength is severely compromised by the unavoidable elastic-plastic transition in the indentation test method. Of course, this challenge has long been recognized in prior literature. As a prime example, Herbert et al. [21] explored several alternatives and could not arrive at any conclusive correlation between the uniaxial yield strength and the indentation yield value. Instead, they simply reported a lower limit and an upper limit based on the theoretical Tresca yield criterion $\left(1.07 \sigma_{y}\right)$ and Tabor's finding $\left(\sim 2.8 \sigma_{y}\right)$, respectively.

A common and standard approach in defining a yield point is to employ an offset strain. Indeed, a tensile or compressive yield strength is often defined as $0.2 \%$ offset yield strength [1]. Close inspection of Figure 3 for the soft and stiff material clearly shows that a fairly large offset is needed if one were to define the indentation yield strength on the plateau region of the ISS curve. However, at the same time, the ISS curves in Figure 6 indicate that the value extracted using a high offset is likely to be strongly influenced by the hardening behavior of the material. So, the practical way forward is some form of a compromise on the offset value.

In order to establish a practically useful offset value of the indentation yield strength, we plotted the offset value indentation stress normalized by the $0.2 \%$ offset uniaxial yield strength for different values of the offset in the ISS curves for the FE simulations performed on the soft material in Figure 9. Based on this plot, it is clear that this ratio exhibits a plateau (more or less constant value) in the range of $0.1 \%-0.2 \%$ offset in the indentation strain. Hence, any offset higher or lower than the range of $0.1 \%-0.2 \%$ offset will result in loss of accuracy in determining the indentation yield strength. Based on the scaling factors we established earlier between the 
indentation plastic strain and the uniaxial plastic strain, a $0.2 \%$ strain offset for the uniaxial test corresponds approximately to $0.15 \%$ offset indentation strain, which falls right in the middle of the plateau seen in the plot in Figure 9. As shown in this figure, the $0.15 \%$ strain offset indentation yield consistently predicts the uniaxial yield strength for a range of hardening behavior, which includes the non-hardening, varying hardening rates, and power law hardening behavior as shown in Figure 9. In other words, this particular offset results in robust estimation of the $0.2 \%$ offset yield strength extracted from the USS curves. With this definition, the scaling factor for the $0.2 \%$ offset yield strength typically recovered from uniaxial stress-strain responses will be 2.0 (see Figure 9). In other words, this would be the constraint factor for the $0.15 \%$ offset definition of the indentation yield point.

\section{Conclusion}

We have addressed the longstanding problem of recovering uniaxial mechanical response from instrumented indentation experiments, with the central challenge being the lack of validated protocols for converting indentation stress and strain values to equivalent values in uniaxial stress states such as simple compression. This study has developed new protocols to address this critical gap for materials exhibiting isotropic plasticity. The protocols presented in this study were validated using FE models of indentation with a broad range of isotropic elastic-plastic responses. Furthermore, the protocols developed involve very simple scaling relationships that are very easy to implement. The overall approach presented in this work has laid a foundation on which one can build an extension for the case of anisotropic plasticity (e.g., crystal plasticity) in the future. 


\section{Acknowledgements}

The authors gratefully acknowledge support from the U.S. Department of Energy, Office of Nuclear Engineering, Nuclear Engineering Enabling Technologies (DOE-NEET). The computations described in this work were partially supported by the Extreme Science and Engineering Discovery Environment (XSEDE), which is supported by National Science Foundation grant number ACI-1053575.

\section{References}

[1] ASTM. Standard Tests Methods for Tension Testing of Metallic Materials. ASTM International. West Conshohocken, PA, 2015.

[2] Uchic MD, Dimiduk DM, Florando JN, Nix WD. Science 2004;305:986.

[3] Shan ZW, Mishra RK, Syed Asif SA, Warren OL, Minor AM. Nat Mater 2008;7:115.

[4] Zhang H, Schuster BE, Wei Q, Ramesh KT. Scripta Materialia 2006;54:181.

[5] Gianola D, Eberl C. JOM 2009;61:24.

[6] Panchal JH, Kalidindi SR, McDowell DL. Computer-Aided Design 2013;45:4.

[7] Roters F, Eisenlohr P, Hantcherli L, Tjahjanto DD, Bieler TR, Raabe D. Acta Materialia 2010;58:1152.

[8] Groh S, Marin EB, Horstemeyer MF, Zbib HM. International Journal of Plasticity 2009;25:1456.

[9] Oliver WC, Pharr GM. Journal of Materials Research 1992;7:1564.

[10] Field J, Swain M. Journal of Materials Research(USA) 1993;8:297.

[11] Vachhani SJ, Doherty RD, Kalidindi SR. Acta Materialia 2013;61:3744.

[12] Pathak S, Kalidindi SR, Klemenz C, Orlovskaya N. Journal of the European Ceramic Society 2008;28:2213.

[13] Pathak S, Stojakovic D, Kalidindi SR. Acta Materialia 2009;57:3020.

[14] Li X, Bhushan B. Materials Characterization 2002;48:11.

[15] Oliver WC, Pethica JB. Method for continuous determination of the elastic stiffness of contact between two bodies. Google Patents, 1989.

[16] Cordill MJ, Moody NR, Gerberich WW. International Journal of Plasticity 2009;25:281.

[17] Pathak S, Kalidindi SR. Materials Science and Engineering: R: Reports 2015;91:1.

[18] Hertz H. Miscellaneous Papers. New York: MacMillan and Co., Ltd., 1896.

[19] Field JS, Swain MV. Journal of Materials Research 1995;10:101.

[20] Swain MV. Materials Science and Engineering A 1998;253:160.

[21] Herbert EG, Pharr GM, Oliver WC, Lucas BN, Hay JL. Thin Solid Films 2001;398-399:331.

[22] Basu S, Moseson A, Barsoum MW. Journal of Materials Research 2006;21:2628.

[23] O'Neill H. Hardness measurement of metals and alloys: Chapman \& Hall, 1967.

[24] Richmond O, Morrison HL, Devenpeck ML. International Journal of Mechanical Sciences 1974;16:75.

[25] Hill R, Storakers B, Zdunek AB. A Theoretical Study of the Brinell Hardness Test, 1989.

[26] Tirupataiah Y, Sundararajan G. Metallurgical Transactions A 1991;22:2375.

[27] Taljat B, Zacharia T, Kosel F. International Journal of Solids and Structures 1998;35:4411.

[28] Beghini M, Bertini L, Fontanari V. International Journal of Solids and Structures 2006;43:2441.

[29] Tabor D. A Simple Theory of Static and Dynamic Hardness, 1948.

[30] Ishlinsky Al. Journal of Applied Mathematics and Mechanics 1944;8:201.

[31] Hill R, Lee EH, Tupper SJ. The Theory of Wedge Indentation of Ductile Materials, 1947.

[32] Yu W, Blanchard JP. Journal of Materials Research 1996;11:2358. 
[33] Bishop RF, Hill R, Mott NF. Proceedings of the Physical Society 1945;57:147.

[34] Johnson KL. Journal of the Mechanics and Physics of Solids 1970;18:115.

[35] Johnson KL. Contact Mechanics: Cambridge University Press, 1987.

[36] Bhattacharya AK, Nix WD. International Journal of Solids and Structures 1988;24:881.

[37] Park YJ, Pharr GM. Thin Solid Films 2004;447-448:246.

[38] Donohue BR, Ambrus A, Kalidindi SR. Acta Materialia 2012;60:3943.

[39] Hay J, Agee P, Herbert E. Experimental Techniques 2010;34:86.

[40] Pelletier H. Tribology International 2006;39:593.

[41] Cheng Y-T, Cheng C-M. International Journal of Solids and Structures 1999;36:1231.

[42] Raabe D, Klose P, Engl B, Imlau K-P, Friedel F, Roters F. Advanced Engineering Materials 2002;4:169.

[43] Bucaille JL, Stauss S, Felder E, Michler J. Acta Materialia 2003;51:1663.

[44] Abu Al-Rub RK, Voyiadjis GZ. International Journal of Plasticity 2004;20:1139.

[45] Liu Y, Varghese S, Ma J, Yoshino M, Lu H, Komanduri R. International Journal of Plasticity 2008;24:1990.

[46] Zhang Jl, Zaefferer S, Raabe D. Materials Science and Engineering: A 2015;636:231.

[47] Reuber C, Eisenlohr P, Roters F, Raabe D. Acta Materialia 2014;71:333.

[48] Patel DK, Al-Harbi HF, Kalidindi SR. Acta Materialia 2014;79:108.

[49] Herrera-Solaz V, LLorca J, Dogan E, Karaman I, Segurado J. International Journal of Plasticity 2014;57:1.

[50] Zaafarani N, Raabe D, Singh RN, Roters F, Zaefferer S. Acta Materialia 2006;54:1863.

[51] Zambaldi C, Raabe D. Acta Materialia 2010;58:3516.

[52] Wang Y, Raabe D, Klüber C, Roters F. Acta Materialia 2004;52:2229.

[53] Demir E, Raabe D, Zaafarani N, Zaefferer S. Acta Materialia 2009;57:559.

[54] Hill R. A Theory of the Yielding and Plastic Flow of Anisotropic Metals, 1948.

[55] ABAQUS. 2014.

[56] Kalidindi SR, Pathak S. Acta Materialia 2008;56:3523.

[57] Tabor D. The Hardness of Metals: ClarendonP, 1951.

[58] Kalpakjian S, Schmid SR. Manufacturing Processes for Engineering Materials: Pearson Education, 2008. 
a)

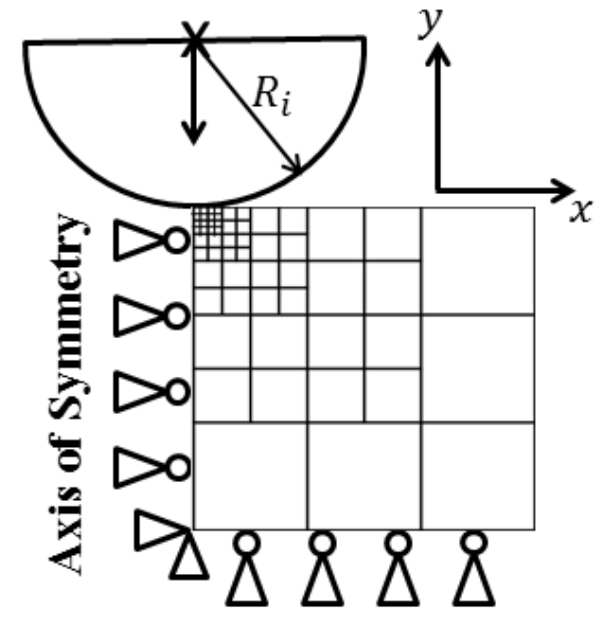

b)

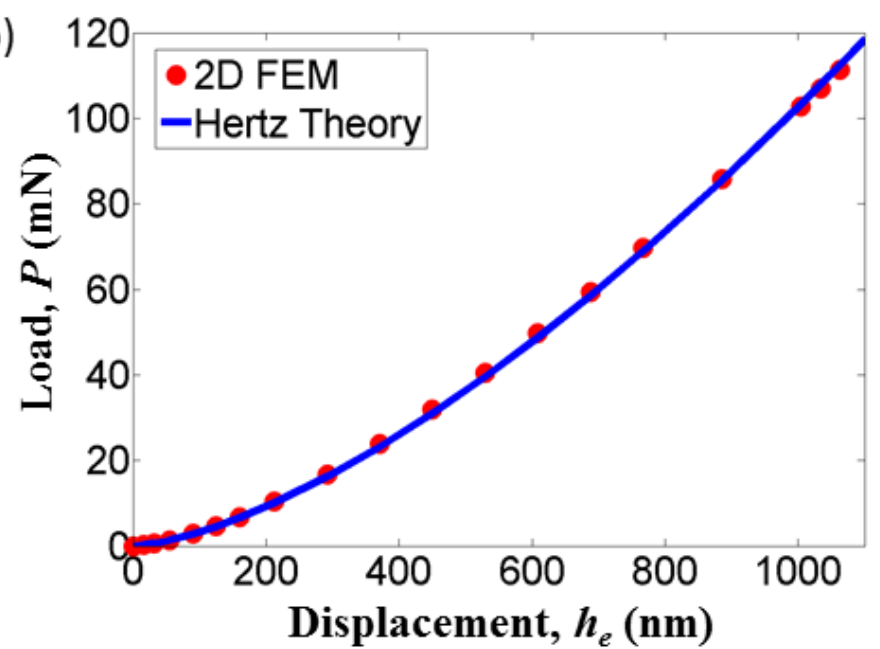

Figure 1. a) Schematic of the two dimensional FE mesh (discretized into 5 regions) to simulate isotropic elastic-plastic response under the indenter (axisymmetric boundary conditions applied). b) Load-displacement response compared from FE simulation against Hertz theory for isotropic elastic response. 


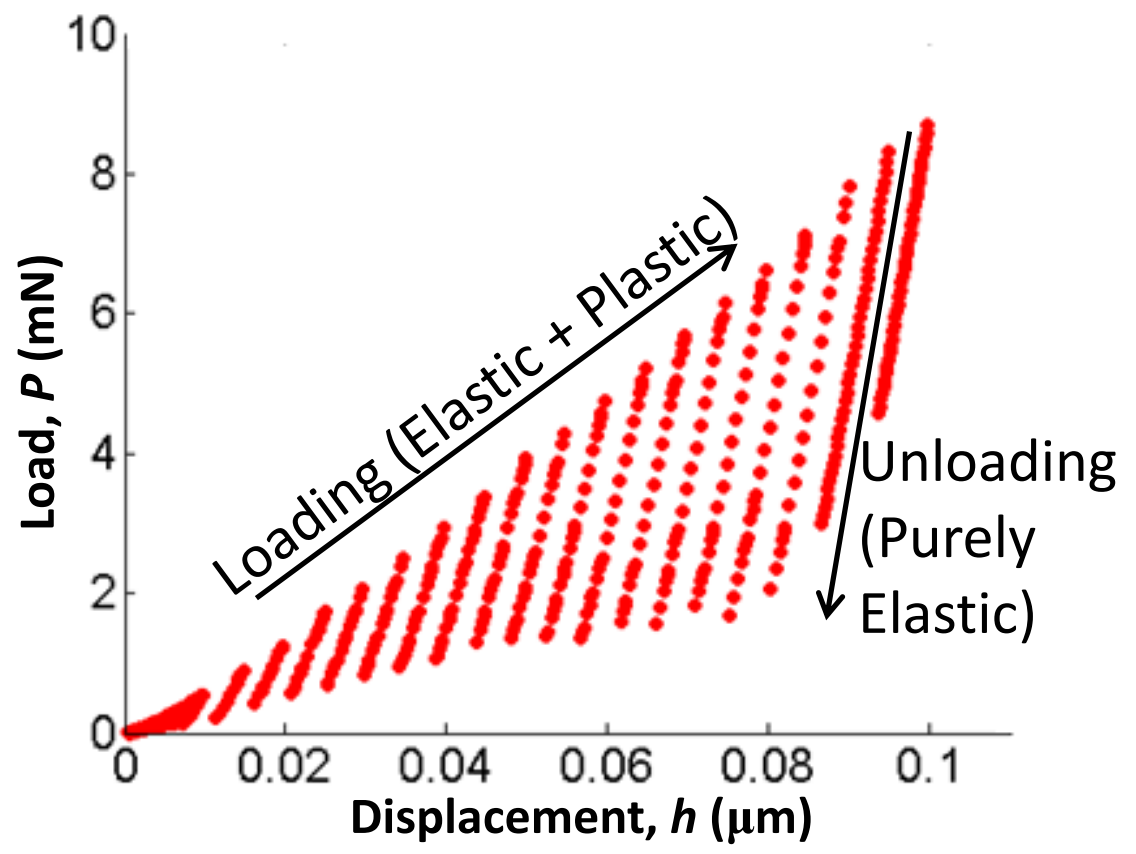

Figure 2. Load-displacement curve depicting partial unloads at multiple load levels. The unloading segments are used to compute indentation stress and strain values. 
a)

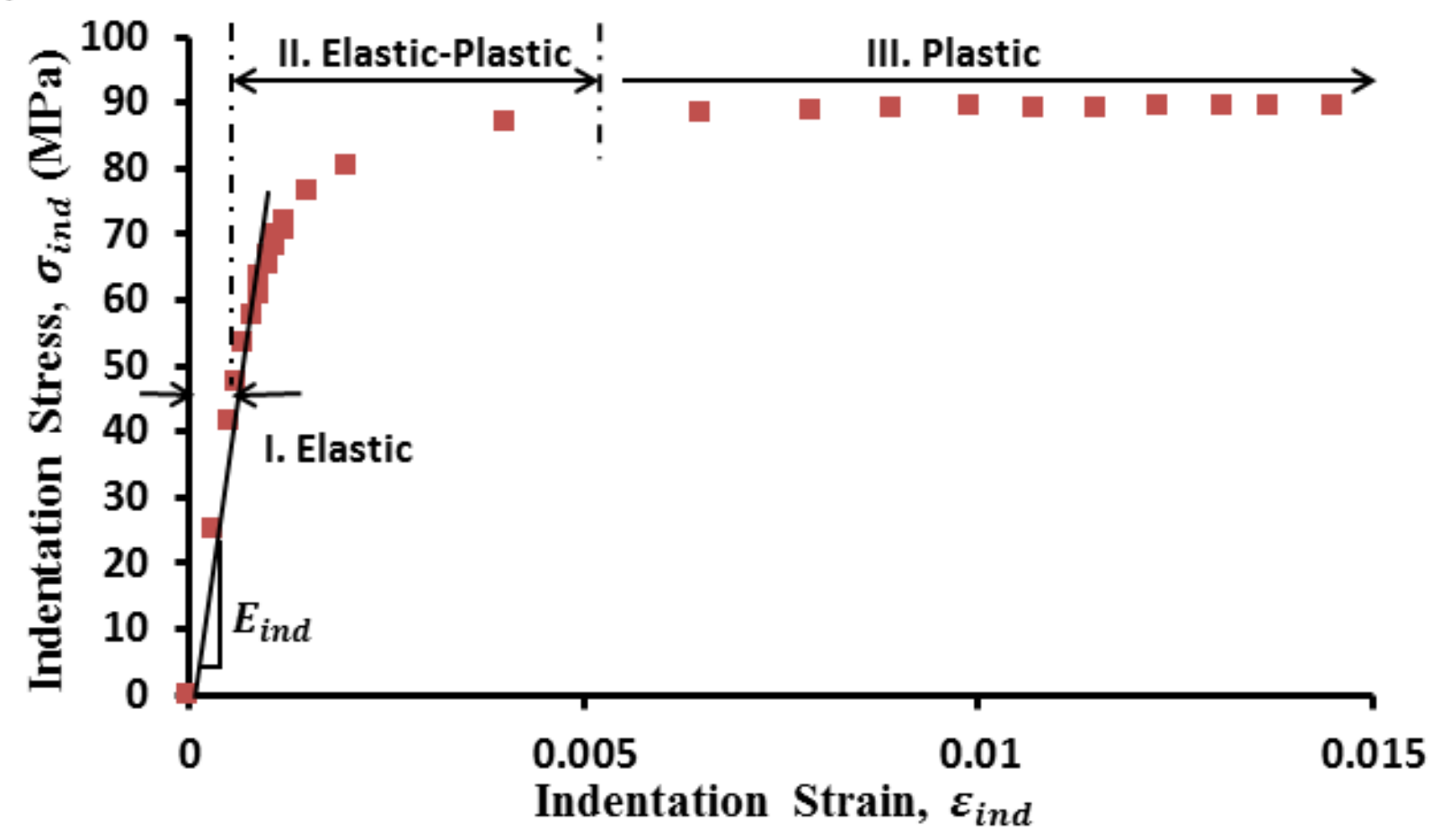

b)

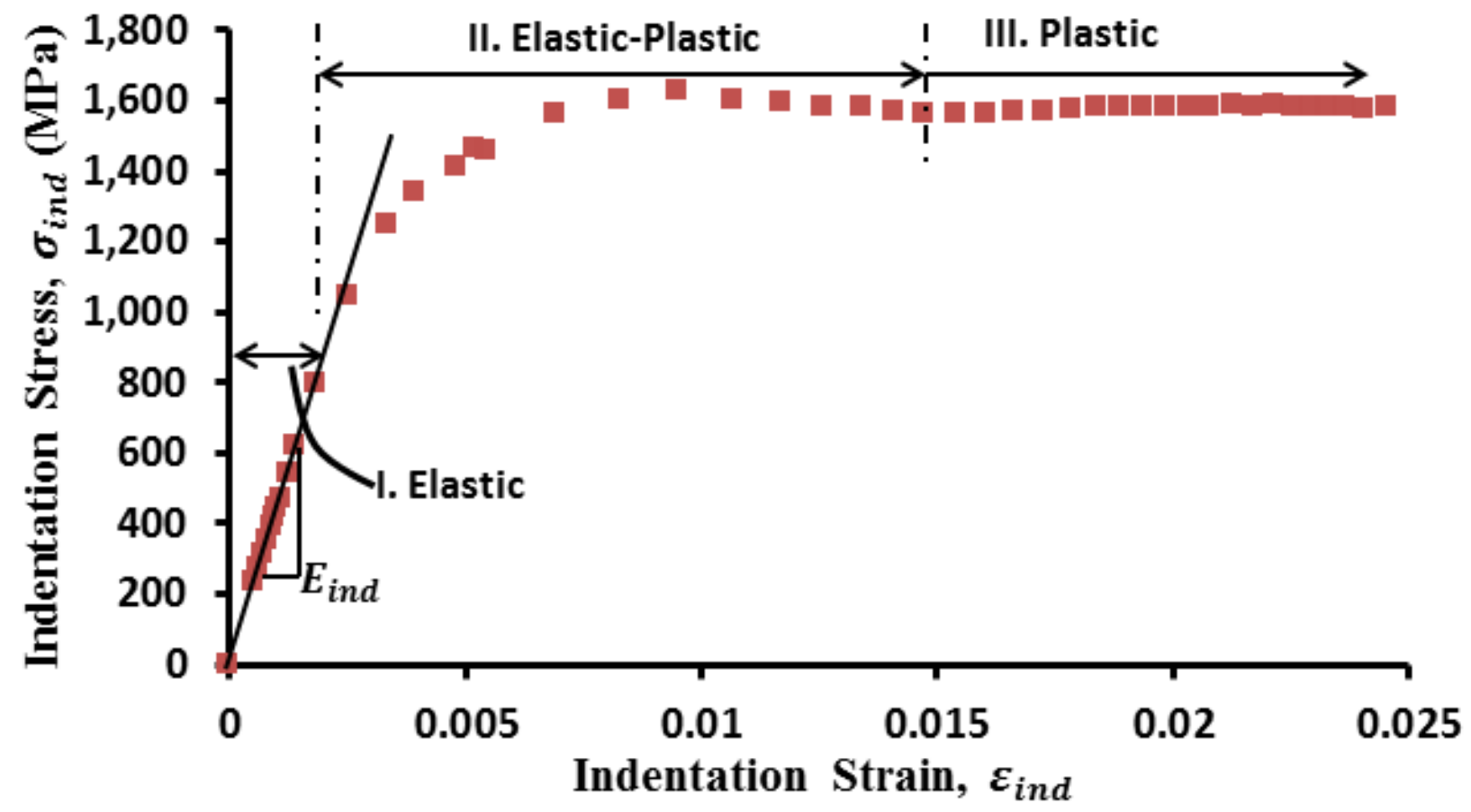

Figure 3. FE-generated indentation stress-strain curves for the a) soft and b) stiff materials selected for this study. 


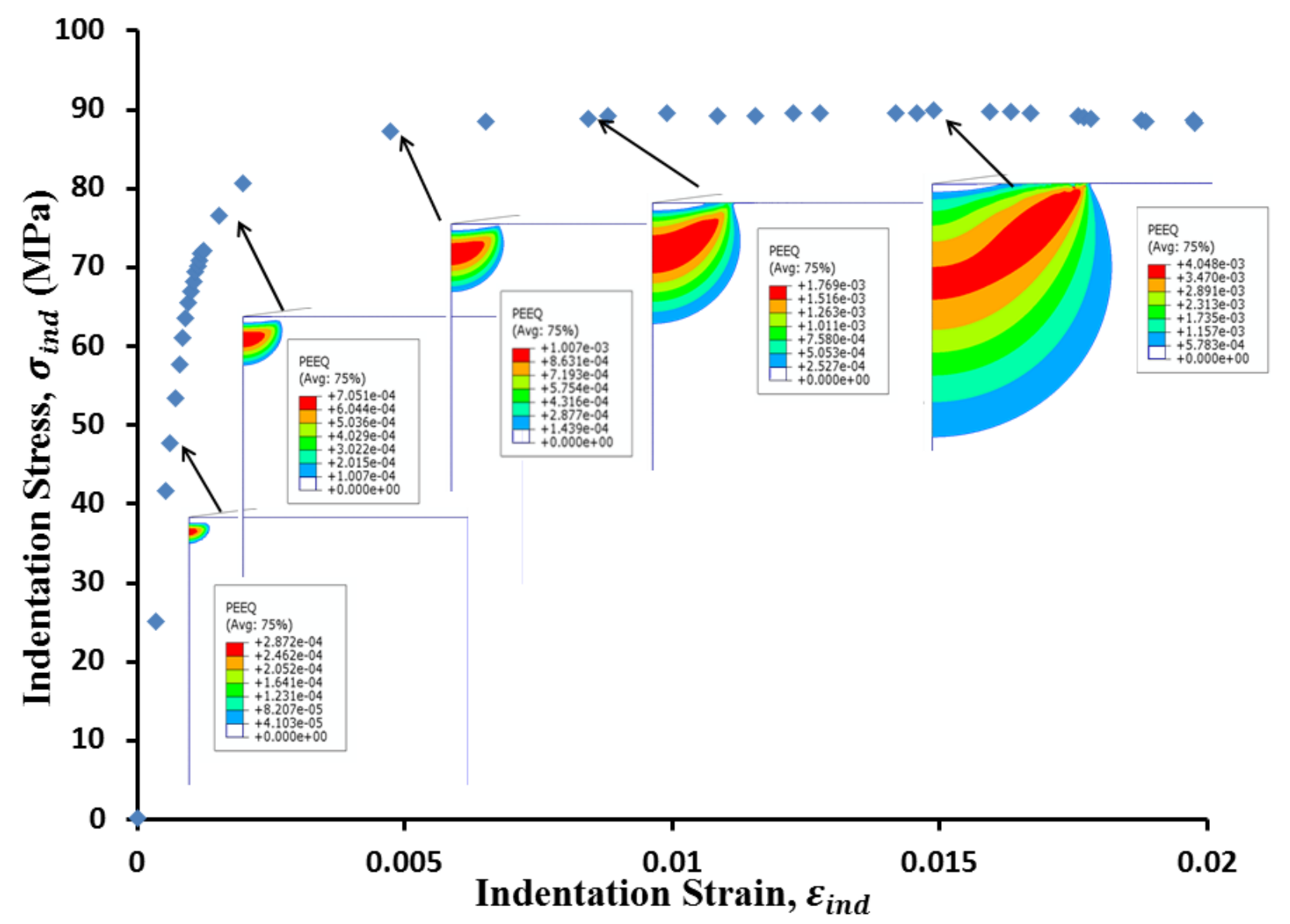

Figure 4. Finite element results showing the evolution of plastic zone under the indenter in terms of contours of equivalent plastic strain field (PEEQ) at the corresponding indentation stress (shown for soft material). Note only small subsection of the mesh is shown here. 


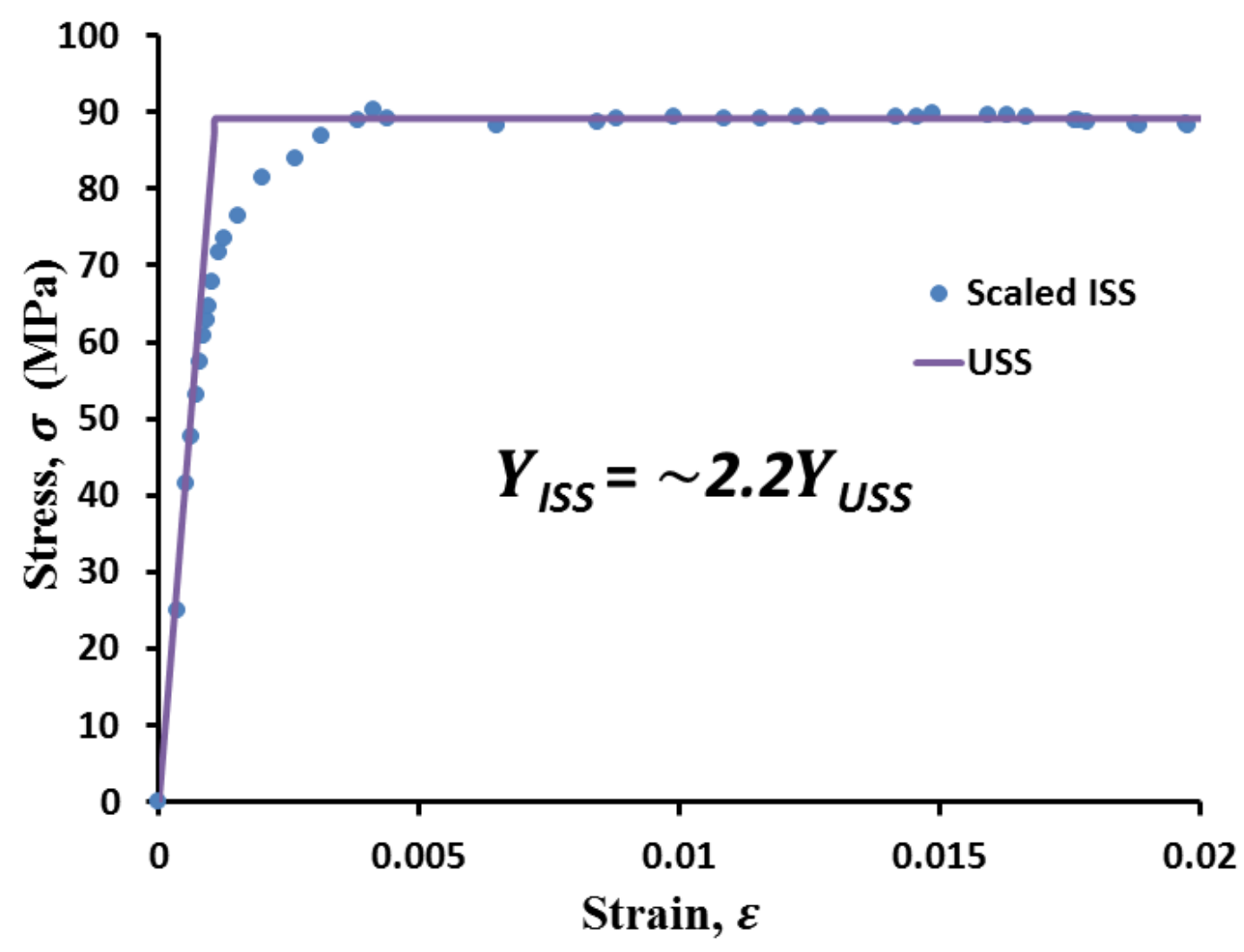

Figure 5. USS curve vs. scaled ISS curve for the soft material (see Table 1) exhibiting elastic perfectly-plastic response. 


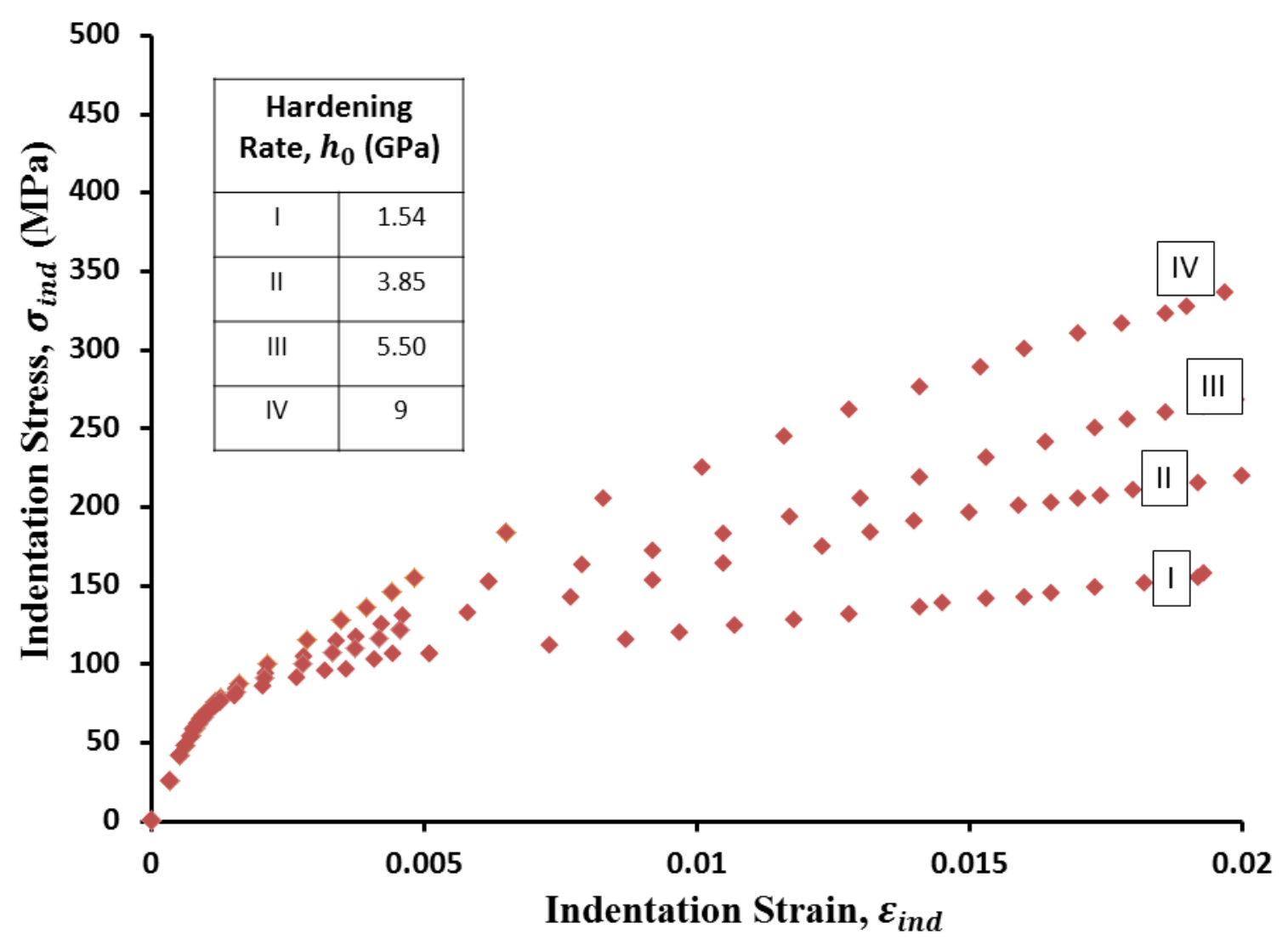

Figure 6. Indentation stress-strain curves for soft material (see Table 1) depicting linearhardening behavior for different hardening rates. 


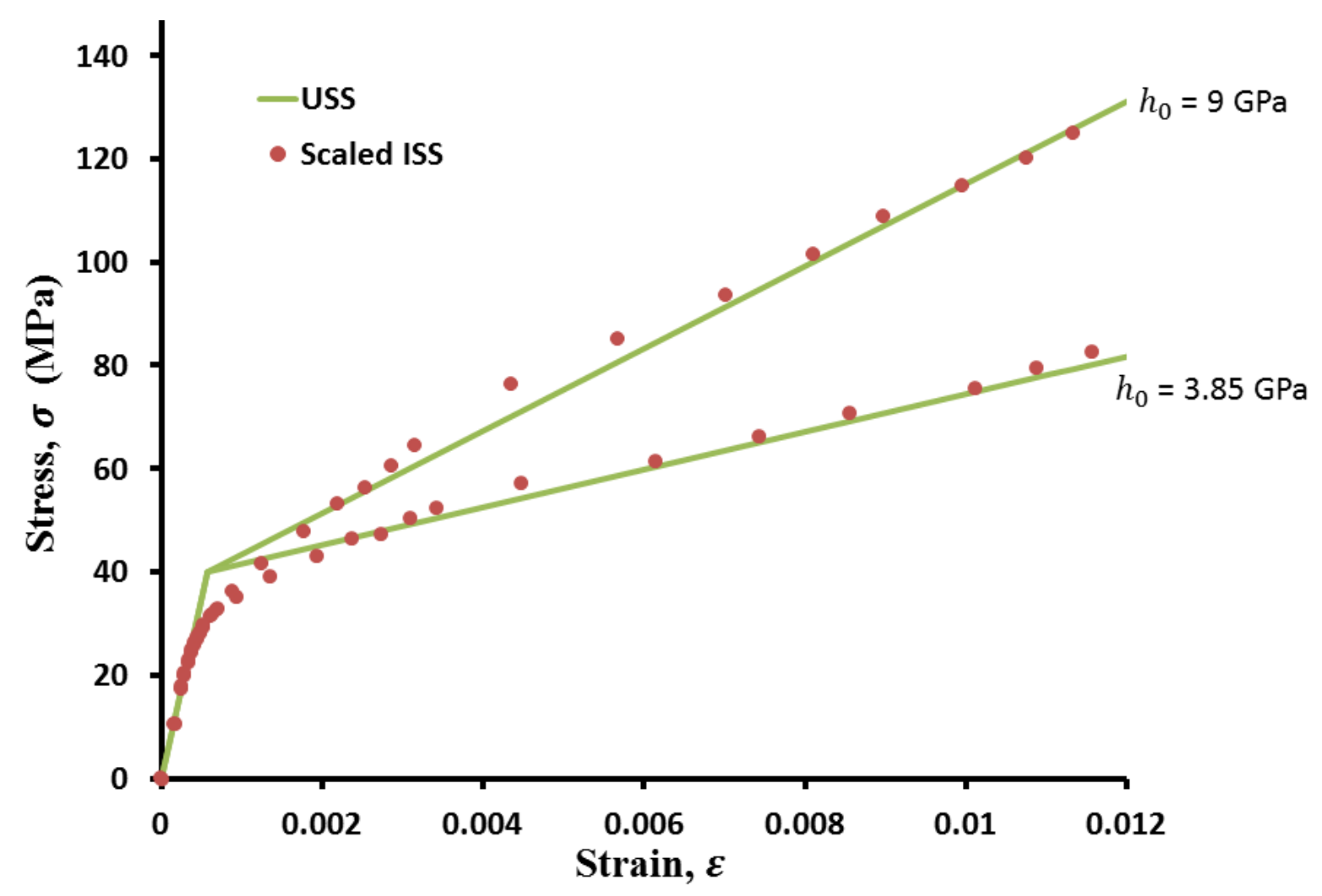

Figure 7. USS curve vs. scaled ISS curve for soft material (see Table 1) with different linear strain hardening rates. 
a)

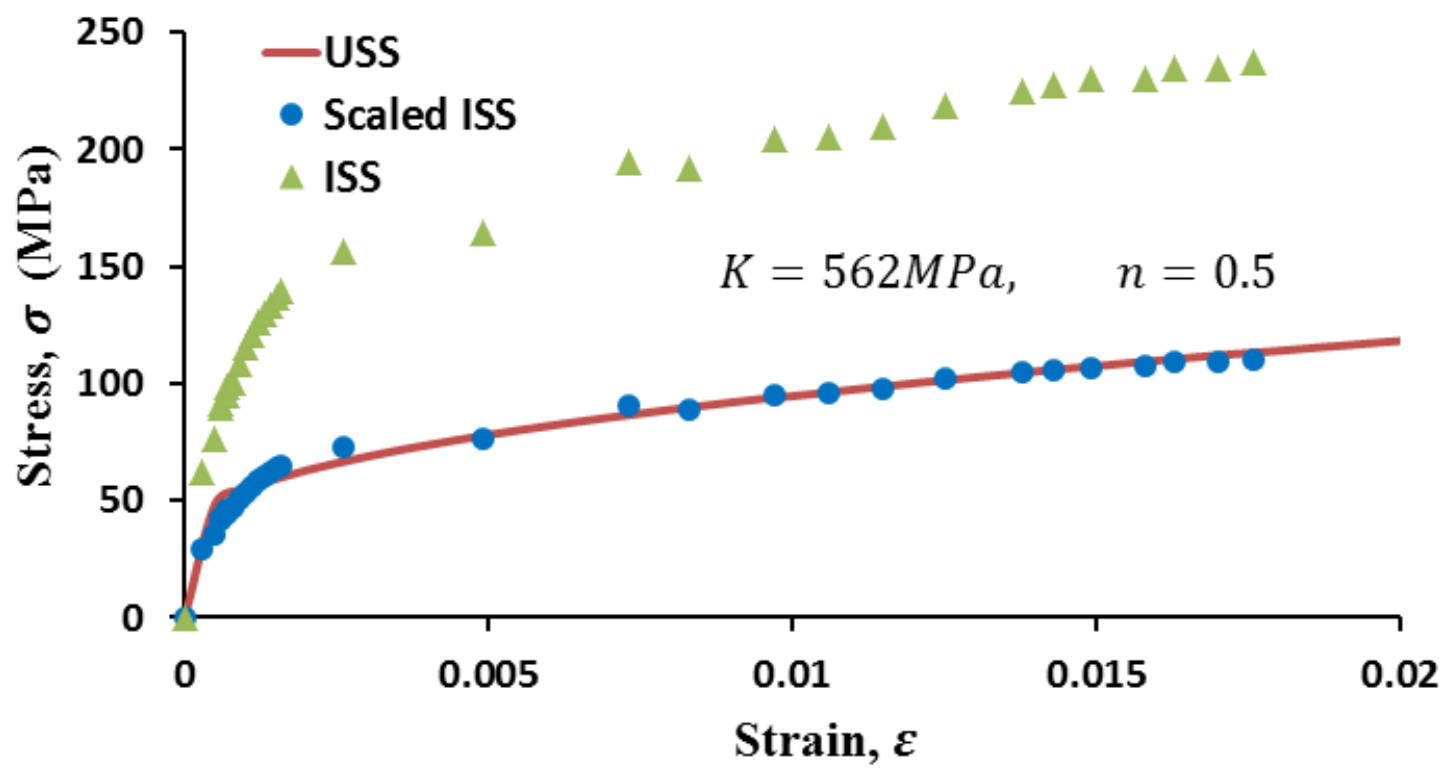

b)

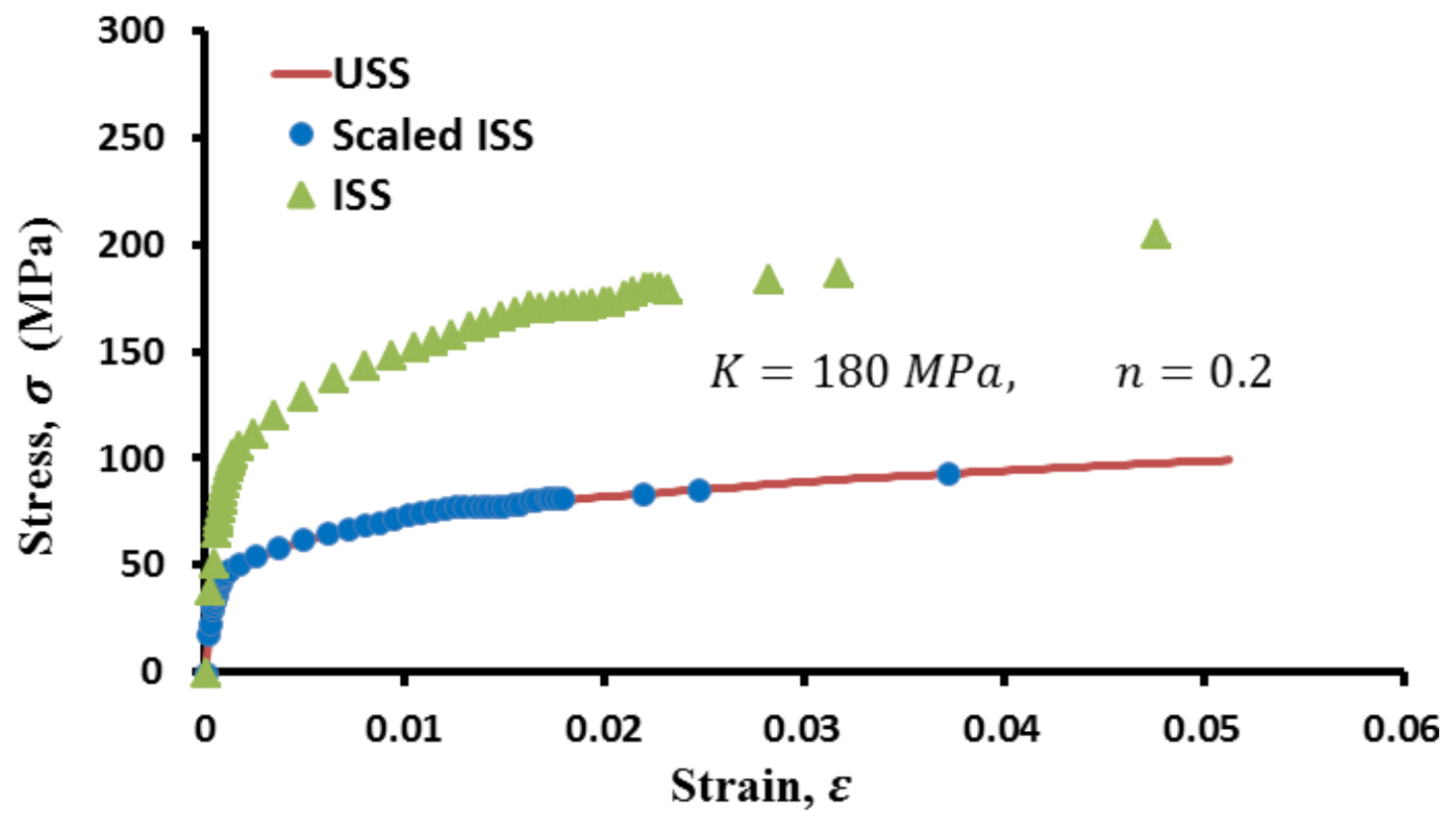

Figure 8. Comparison of the ISS, Scaled ISS, and USS responses for materials exhibiting power-law hardening behavior. 


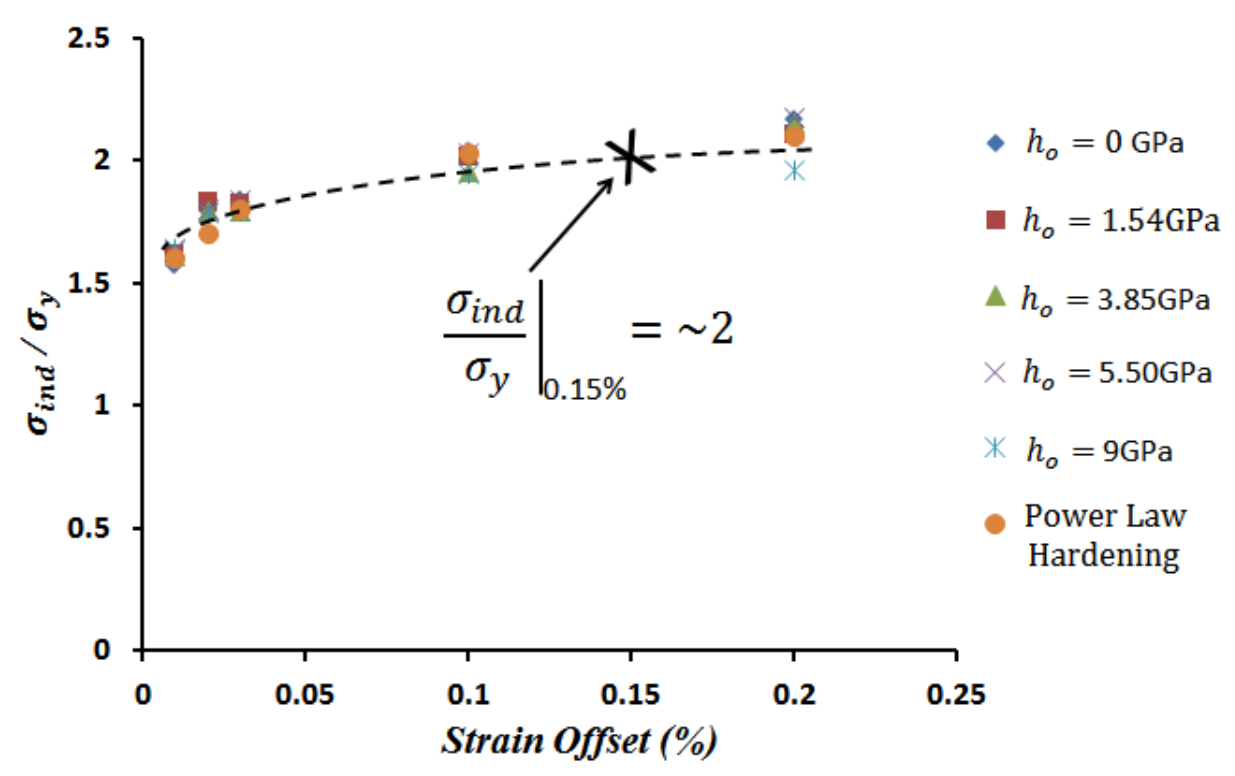

Figure 9. Ratio of indentation stress to the $0.2 \%$ offset uniaxial yield strength for all FE simulations performed on the soft material. 


\begin{tabular}{|c|c|c|c|}
\hline Material & $\begin{array}{c}\text { Young's Modulus } \\
\text { (GPa) }\end{array}$ & $\begin{array}{c}\text { Poisson's } \\
\text { Ratio }\end{array}$ & $\begin{array}{c}\text { Yield Strength } \\
\text { (GPa) }\end{array}$ \\
\hline Soft & 70 & 0.30 & 0.04 \\
\hline Stiff & 400 & 0.28 & 0.75 \\
\hline
\end{tabular}

Table 1. Elastic and plastic properties used to describe the isotropic sample material behavior used in the present study. 
Protocols to Correlate Indentation Stress-Strain (ISS) to Uniaxial Stress-Strain (USS)

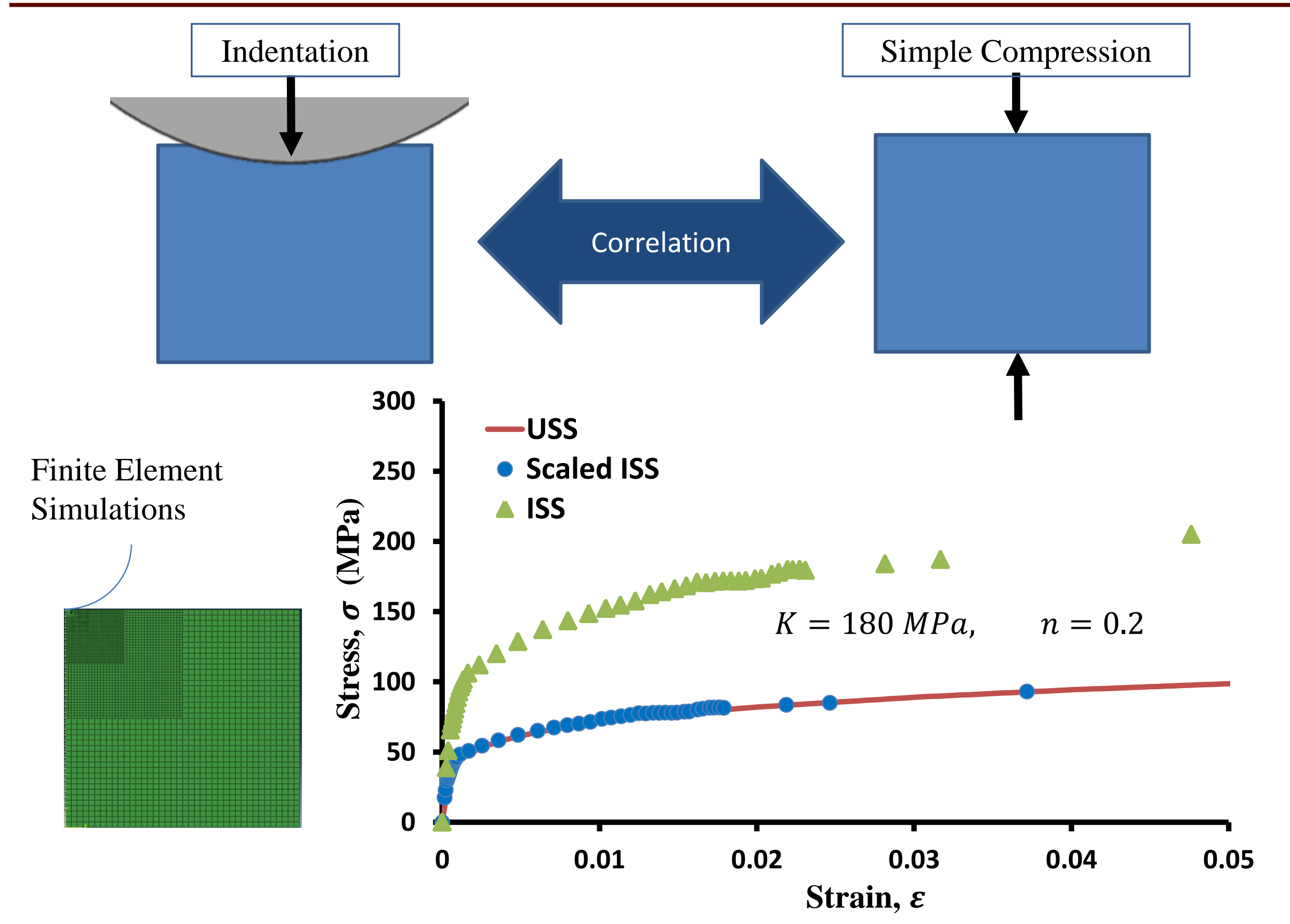

